\title{
Mapping of Two Dimensional Electron Gas at Atomically Abrupt Oxide Interfaces using Inline Electron Holography
}

Kyung Song ${ }^{1}$, Christoph T. Koch ${ }^{2}$, Si-Young Choi ${ }^{3}$, Ho Nyung Lee ${ }^{4}$, Sangwoo Ryu ${ }^{5}$, Chang-Beom Eom $^{5}$ and Sang Ho $\mathrm{Oh}^{1}$

1. Department of Materials Science and Engineering, Pohang University of Science and Technology (POSTECH), San 31, Hyoja-dong, Nam-gu, Pohang 790-784, Republic of Korea

2. Department of Physics, Ulm University, Albert-Einstein-Allee 11, 89081 Ulm, Germany

3. Advanced Characterization and Analysis Group, Korea Institute of Materials Science (KIMS), Changwon 642-831, Republic of Korea

4. Materials Science \& Technology Division, Oak Ridge National Laboratory, Oak Ridge, TN 37831, USA

${ }^{5 .}$ Department of Materials Science and Engineering, University of Wisconsin-Madison, Madison, WI 53706, USA

Two-dimensional conducting layer at the interface between the insulating perovskite oxides has been recently discovered by virtue of the state-of-art growth technique, which is able to control the single atomic layer and thus generate the creation of atomically-abrupt interfaces even in the complex oxide materials [1]. At this atomically-controlled interface, the carrier concentration can be altered with the polarity induced electric field [2], and also the conducting layer can apparently be localized within a few $\mathrm{nm}$ of the interface, resulting in a two-dimensional electron gas (2DEG) at the interface. 2DEG, which is profoundly related with the electronic reconstruction, provides the superconductivity along the interface even between two insulating perovsikte oxides. A compelling issue related to this interface material is the spatial distribution of the interfacial electric potential, and its influence on the interfacial charge density. While, up to date, 2DEG has been studied in an indirect way, which can be achieved via electron energy loss spectroscopy (EELS), we focus on $\mathrm{m}$ ore direct information on electronic reconstruction of the electric potential at and near the hetero-interface.

In this study, we attempted to quantitatively interpret the 2DEGs forming at the proven oxide heterostructures such as $\mathrm{LaAlO}_{3} / \mathrm{SrTiO}_{3}$ (LAO/STO) and $\mathrm{LaTiO}_{3} / \mathrm{SrTiO}_{3}$ (LTO/STO) using inline electron holography. The electrostatic potential distribution across the interfaces could be feasibly extracted from the phase information from a series of through-focal bright-field TEM images (Fig. 1) [3]. Note that the as-reconstructed potential map includes not only the electrostatic potential generated by static charges (including 2DEGs) but also the background potential, i.e. mean inner potential. Assuming that the difference in mean inner potential between two oxide materials is not significant, the obtained potential can be treated to obey the Poisson's equation. Therefore, the total charge density across oxide hetero-interfaces can be obtained by applying a Laplacian image filter to the obtained potential map.

The charge density map revealed asymmetric distribution with different values at the lower LTO/STO interfaces and the upper STO/LTO interfaces (Fig. 2). The 2DEG-like free electrons were confined only to the lower LTO/STO interfaces with a density of $\sim 3 \times 10^{14} \mathrm{~cm}^{-2}$, which agrees well with the transfer of 0.5 electron per a unit cell of $\mathrm{LaTiO}_{3}$ to the adjacent $\mathrm{SrTiO}_{3}$ layers. On the other hand, the upper interfaces were depleted of electrons, which is analogous to acceptor doping. The atomic scale Zcontract STEM images revealed that the upper STO/LTO interfaces are more diffusely rough in contrast 
to the automatically smooth LTO/STO lower interfaces, indicating that the interface roughness might have an effect on the formation of 2DEGs in the perovskite heterostructure.

References:

[1] A. Ohtomo and H. Y. Hwang, Nature 427 (2004), 423.

[2] S. Thiel et al, Science 313 (2006), 1942.

[3] C. T. Koch, European Microscopy Congress EMC2012 (2012) 139.

[4] The authors acknowledge funding from the Basic Science Research Program through the National Research Foundation of Korea (NRF) funded by the Ministry of Education, Science and Technology (Grant 2010-0005834, 2011-0029406).
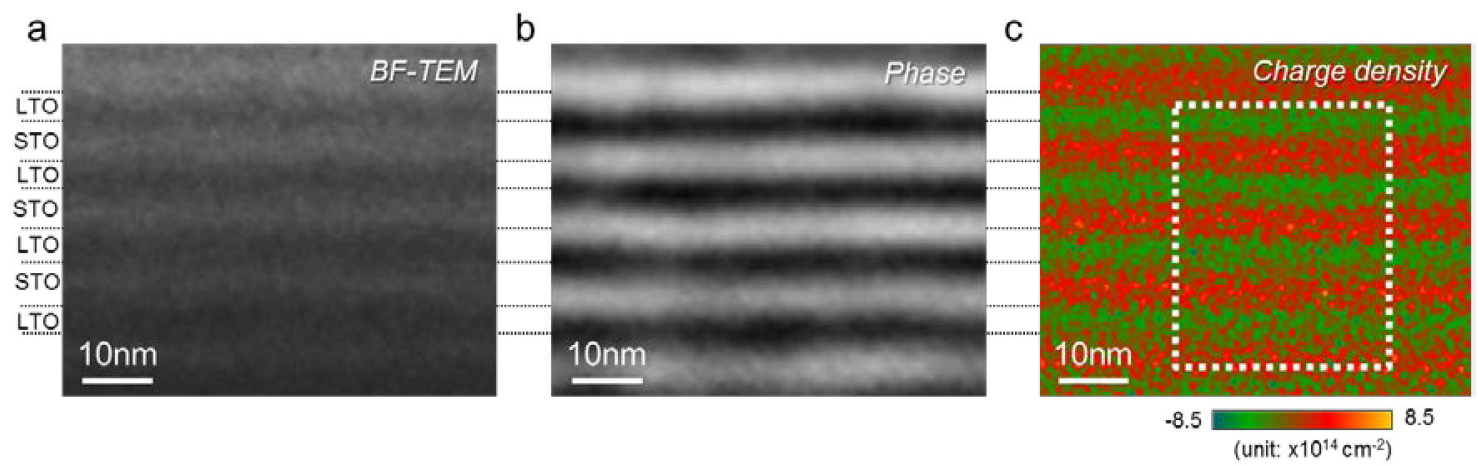

Figure 1. a. Bright-field TEM image, b. Reconstructed phase map and, c. charge density map of the LTO/STO heterostructure.

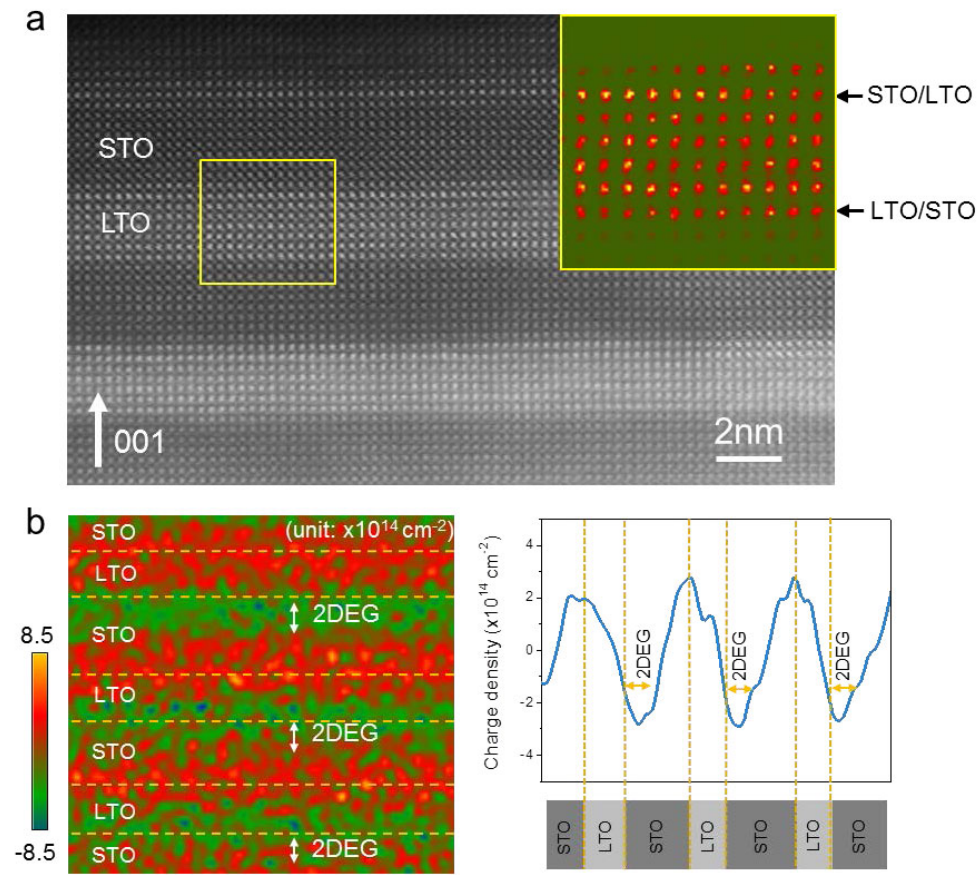

Figure 2. a. STEM Z-contrast image. The inset highlights the different interface roughness at the two interfaces. b. Magnified view of the charge density map and a profile across the LTO/STO heterostructure. The 2DEGs formed at the lower LTO/STO interfaces are marked by arrows. The LTO and STO are 6 and 9 unit-cell thick, respectively. 